\title{
Percepción de bullying en alumnos de sexto año de la escuela de medicina de una universidad pública de Lima 2015
}

Perception of bullying in sixth year students of a Lima public university school of

medicine 2015

\author{
Miguel Oliveros Donohue ${ }^{1, a}$, Alberto Perales Cabrera ${ }^{1, a}$, Salomón Zavala Sarrio,a, \\ Isabel Amemiya Hoshi ${ }^{1, b}$, Miguel Angel Pinto Salinas ${ }^{1, c, d}$, Anthony Jazet Ramos \\ Yataco $^{1,0}$ \\ Facultad de Medicina, Universidad Nacional Mayor de San Marcos, Lima, Perú.

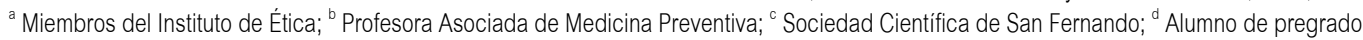

\begin{abstract}
Resumen
Introducción. Las conductas agresivas y discriminatorias afectan la salud física y mental incluso de quienes las cometen. Los estudiantes están sometidos a situaciones de estrés, las cuales aumentan el riesgo de desarrollar dichas conductas y a sufrir sus consecuencias. Por otro lado, la Escuela de Medicina tiene como propósito formar integralmente profesionales médicos, con capacidad de evitar situaciones de violencia y convivir pacíficamente. Es por ello el interés de describir este problema en la Escuela de Medicina de la Universidad Nacional Mayor de San Marcos, Lima, Perú. Objetivo. Abordar la situación de violencia (bullying) en estudiantes de Medicina Humana del $6^{\circ}$ año que no habian participado en el Programa de Formación Integral en la UNMSM. Diseño. Estudio descriptivo-transversal. Lugar. Sede docente "Instituto Nacional de Salud del Niño", Lima, Perú. Participantes. Muestra conformada por $93(63 \%)$ estudiantes matriculados en el sexto año de la Facultad de Medicina que llevaron el curso de Pediatría durante los meses de julio a noviembre de 2015. Intervenciones. Se utilizó un instrumento desarrollado y validado por la Defensoría del Pueblo de España, modificado y corregido por Hoyos y col. Se calcularon las frecuencias y porcentajes de las respuestas. Resultados. Las conductas de violencia fueron más prevalentes entre estudiantes, entre ellas la conducta de agresión verbal más practicada fue la de expresarse de mala manera $(40,8 \%)$. Conclusiones. La existencia de violencia se presenta en un alto porcentaje de la población estudiada y están implicados en ella docentes y estudiantes.
\end{abstract}

Palabras clave. Violencia; Bullying; Estudiantes; Docentes.

\section{Abstract}

Introduction. Aggressive and discriminatory behavior affects physical and mental health even on those who commit them. Students are subjected to stress, which increases the risk of those behaviors and makes them likely to suffer from its consequences. On the other hand, the Medical School aims to educate medical professionals integrally, with abilities to avoid violence and live peacefully. That is why the interest of describing this problem in Universidad Nacional Mayor de San Marcos, Lima, Peru's Medical School. Objective. To address violence in students from the sixth year of Human Medicine who had not participated in the Integral Formation Program in UNMSM. Design: Descriptive, transversal study. Setting. "Instituto Nacional del Niño", Lima, Peru, teaching hospital. Participants. Sample consisted in $93(63 \%)$ students enrolled in the sixth year of the Faculty of Medicine who took the course of Pediatrics during the months of July to November 2015. Interventions. An instrument developed and validated by the Spanish Office of the Ombudsman, modified and corrected by Hoyos et al was used. Frequencies and percentages of responses were calculated. Results. Violent behaviors were more prevalent among students; the most practiced behavior was to express badly (40.8\%). Conclusions. The existence of violence occurs in a high percentage of the studied population; teachers and students are involved.

Keywords. Violence; Bullying; Stress; Students; Teachers.

An Fac med. 2016;77(3):231-6 / http://dx.doi.org/10.15381/anales.v77i3.12406

\section{INTRODUCCIÓN}

La Escuela de Medicina tiene como misión formar buenos ciudadanos y brindarles educación para que, ejerciendo un trabajo profesional serio y responsable, alcancen un futuro digno con educación en valores, capacidad para ejercer éticamente deberes y derechos, y respeto a los demás, aprendiendo a convivir pacíficamente. De esta manera, se complementa y fortalece la educación que se inició en el hogar, luego en las aulas, y se ha continuado con el régimen de tutoría universitaria. La educación en la niñez y juventud debe ser compartida por estos dos estamentos socializadores, hogar y escuela, los que, acrecentados con la tutoría en pregrado, nos permitirá obtener futuros médicos con formación integral ${ }^{(1,2)}$.
Lamentablemente, con frecuencia se aprecia en la escuela primaria y secundaria conductas que implican agresiones verbales o físicas entre los estudiantes, faltas de respeto, marginación o acoso que causan temor, llevando incluso al abandono escolar. Las consecuencias de una conducta agresiva y discriminatoria sobre las personas, e incluso sobre quienes las ejecutan, son 
muy graves, más aún cuando ocurren a edad temprana, dejando secuelas para toda la vida (2). Estar involucrado en situaciones de maltrato conduce con frecuencia al desarrollo de tendencias violentas, delincuencia y criminalidad en la vida adulta, mostrando conductas antisociales asociadas con el consumo de drogas, alcohol, robos, mala adaptación escolar y riesgo de fracaso ${ }^{(3-5)}$.

La violencia escolar (bullying) ha sido estudiada tanto en primaria como en secundaria; sin embargo, no hay muchas investigaciones a nivel superior en instituciones y universidades de América Latina, excepto en Colombia, Chile, y recientemente Perú ${ }^{(6-9)}$. Se han realizado diversas publicaciones sobre su presentación durante el desarrollo de la educación médica en Estados Unidos y Europa ${ }^{(4,10-13)}$, y fue considerada por algunos investigadores como la principal fuente de estrés entre los estudiantes de medicina ${ }^{(14-16)}$.

Por otro lado, es necesario aclarar que si bien el bullying supone una relación de poder dialéctico entre víctima y victimario, los cuales se encuentran bajo la misma condición (de iguales), algunos autores asumen que una modalidad de la intimidación es la que se puede denominar acoso docente o bullying vertical, escondido en muchas ocasiones por ese ejercicio de autoridad ${ }^{(17,18)}$. Así, algunos de los comportamientos de los docentes pueden tener carácter intimidatorio, en relación con el estrés del ambiente donde se labora, especialmente con alumnos que están finalizando la carrera, quienes ya tienen responsabilidades médicas ${ }^{(11)}$.

La Universidad Nacional Mayor de San Marcos (UNMSM) ha desarrollado desde el 2014, un Programa de Formación Integral de los alumnos que ingresan a la Facultad de Medicina, y un Programa de Formación de Tutores, poniendo énfasis en las cinco dimensiones del desarrollo humano: material, intelectual, emocional, trascendental y social. En esta oportunidad, nos interesaba conocer este problema en la Escuela de Medicina de la UNMSM con estudiantes que antecedieron a la formación integral ${ }^{(19)}$.

El objetivo fue abordar la situación de la violencia (bullying) en estudiantes de Medicina Humana del $6^{\circ}$ año que no habían participado en el Programa de Formación Integral en la UNMSM. Adicionalmente, determinar la frecuencia de percepción de violencia verbal, física, de exclusión social, maltrato de docentes, entre iguales, comportamiento sexual y describir las manifestaciones del maltrato en función de la edad, género de víctimas y agresores. Identificar a quién comunica la violencia percibida y describir el comportamiento de los compañeros que presenciaron la agresión.

\section{MÉTODOS}

Se aplicó un diseño transversal descriptivo para evaluar la percepción de violencia (bullying) en nuestra Escuela de Medicina. La población estuvo conformada por 147 estudiantes matriculados en el $6^{\circ}$ año de la Facultad de Medicina (87 varones y 60 mujeres), que llevaron el curso de Pediatría durante los meses de julio a noviembre de 2015 en las aulas del Instituto Nacional de Salud del Niño. La muestra la conformaron 93 (63\%) de dichos estudiantes.

Se utilizó un instrumento desarrollado y validado en España por la Defensoría del Pueblo, modificado, corregido y validado por Hoyos y col ${ }^{(6,18)}$. El instrumento que hemos empleado no ha sido modificado; cuatro de las pregun- tas se refieren a estudiantes y docentes; las restantes se han dedicado a los estudiantes, diferenciando el género y la participación en los actos de violencia como víctimas, agresores o testigos.

Los participantes aceptaron participar en la investigación dando un consentimiento informado oral. Se dejó abierta la posibilidad de rechazo. La encuesta fue anónima. Tuvieron la oportunidad de elegir las preguntas a contestar y de retirarse de la investigación en cualquier momento. Se les aseguró la confidencialidad de la información a quienes escribieron su correo electrónico en la encuesta. Los resultados de la investigación les fueron comunicados y publicados sin revelar ninguna identificación personal. Esta investigación contó con la aprobación del Comité de Ética de la Facultad de Medicina de la UNMSM.

\section{RESULTADOS}

\section{PERCEPCIÓN DE CONDUCTAS DE AGRESIÓN VERBAL}

Las conductas de agresión verbal que predominaron entre los estudiantes fueron: expresarse de mala manera, poner apodos e insultos y contestar de manera desafiante. En los estudiantes, al referirse a los docentes, predominaron la conducta desafiante y apodos. En la conducta de los docentes hacia los estudiantes, primó igualmente las respuestas desafiantes, apodos e insultos (tabla 1).

Tabla 1. Percepción de conductas de agresión verbal $(n=93)$.

\begin{tabular}{|c|c|c|c|c|c|c|}
\hline \multirow{2}{*}{ Tipo de agresión verbal } & \multicolumn{2}{|c|}{ Grupo A } & \multicolumn{2}{|c|}{ Grupo B } & \multicolumn{2}{|c|}{ Grupo C } \\
\hline & $\mathrm{n}^{\circ}$ & $\%$ & $\mathrm{n}^{\circ}$ & $\%$ & $\mathrm{n}^{\circ}$ & $\%$ \\
\hline Insultar & 34 & 36,5 & 9 & 9,6 & 16 & 17,2 \\
\hline Contestar desafiante & 30 & 32,2 & 18 & 19,3 & 25 & 26,9 \\
\hline Poner apodos & 52 & 55,0 & 18 & 19,3 & 17 & 18,3 \\
\hline Hablar mal & 38 & 40,8 & 12 & 12,9 & 15 & 16,1 \\
\hline Dibujos ofensivos & 3 & 3,20 & 3 & 3,20 & 5 & 5,4 \\
\hline
\end{tabular}

Grupo A: Entre estudiantes. Grupo B: De estudiantes a docentes. Grupo C: De docentes a estudiantes. 
Tabla 2. Percepción de conductas de agresión por exclusión.

\begin{tabular}{|c|c|c|c|c|c|c|}
\hline \multirow{2}{*}{ Tipo de agresión por exclusión } & \multicolumn{2}{|c|}{ Grupo A } & \multicolumn{2}{|c|}{ Grupo B } & \multicolumn{2}{|c|}{ Grupo C } \\
\hline & $\mathrm{n}^{\circ}$ & $\%$ & $\mathrm{n}^{\circ}$ & $\%$ & $\mathrm{n}^{\circ}$ & $\%$ \\
\hline No permitir participación en grupos & 33 & 35 & 7 & 8,0 & 9 & 10,0 \\
\hline Infringir reglas del grupo & 18 & 19 & 5 & 5,0 & 7 & 7,0 \\
\hline Impedir realizar actividades & 14 & 15 & 3 & 3,0 & 9 & 10,0 \\
\hline No permitir acceso a la información & 21 & 22 & 6 & 6,0 & 12 & 13,0 \\
\hline Ignorar & 35 & 38 & 7 & 8,0 & 18 & 19,0 \\
\hline
\end{tabular}

Grupo A: Entre estudiantes. Grupo B: De estudiantes a docentes.

Grupo C: De docentes a estudiantes.

Tabla 3. Percepción de conductas de agresión física ( $n=93)$.

\begin{tabular}{|c|c|c|c|c|c|c|}
\hline \multirow{2}{*}{ Tipo de agresión física } & \multicolumn{2}{|c|}{ Grupo A } & \multicolumn{2}{|c|}{ Grupo B } & \multicolumn{2}{|c|}{ Grupo C } \\
\hline & $\mathrm{n}^{\circ}$ & $\%$ & $\mathrm{n}^{\circ}$ & $\%$ & $\mathrm{n}^{\circ}$ & $\%$ \\
\hline Golpear & 31 & 33 & 4 & 4,0 & 7 & 7,0 \\
\hline Empujar & 33 & 35 & 4 & 4,0 & 11 & 12,0 \\
\hline No permitir acceso a objetos & 16 & 17 & 2 & 2,0 & 10 & 11,0 \\
\hline Esconder las cosas & 16 & 17 & 3 & 3,0 & - & - \\
\hline Destruir objetos & 12 & 13 & 1 & 1,0 & 1 & 1,0 \\
\hline Apropiarse objetos & 15 & 16 & 1 & 1,0 & 1 & 1,0 \\
\hline
\end{tabular}

Grupo A: Entre estudiantes. Grupo B: De estudiantes a docentes.

Grupo C: De docentes a estudiantes.

\section{PERCEPCIÓN DE CONDUCTAS DE AGRESIÓN POR EXCLUSIÓN SOCIAL}

Entre estudiantes, la agresión por exclusión social se manifestó en una conducta caracterizada por no permitir la participación en grupos, así como ignorar a sus compañeros. En la conducta de ellos con sus docentes, tuvieron una distribución de conducta similar pero con menor frecuencia.

Los docentes preferentemente ignoraban a sus estudiantes y no les permitían acceso a la información (tabla 2).

\section{PERCEPCIÓN DE CONDUCTAS DE AGRESIÓN FÍSICA}

La agresión física entre estudiantes se expresó sobre todo con golpes y empujones, pero también se manifestó con esconder las cosas, destruir y apropiarse de objetos. La agresión física de estudiantes con docentes fue escasa y se manifestó con golpes y empujones. La agresión de los docentes se percibió con más frecuencia por los estudiantes, no permitiendo acceso a objetos, incluyendo hasta golpes y empujones (tabla 3).

\section{PERCEPCIÓN DE MANIFESTACIONES DE MALTRATO ENTRE IGUALES}

La violencia ejemplificada por el maltrato entre iguales (bullying), al calificar las respuestas, mostró evidencias de maltrato físico, psicológico, social y sexual. Los testigos cobran importancia al no estar implicados directamente en el maltrato. Dentro de este grupo, en orden descendente, predominaron las variables hablar mal de él o ella, poner apodos, ignorar, insultar y no dejar participar (tabla 4).

\section{TIPO DE MALTRATO EN FUNCIÓN DE GÉNERO DE VÍCTIMAS Y AGRESORES}

La respuesta me ignoran, hablan mal de mí y me ponen apodos fueron más frecuentes en víctimas del género masculino, mientras que en las víctimas femeninas, me ponen apodos y hablan mal de mí fueron las respuestas más frecuentes (tabla 5).

Las respuestas de las víctimas masculinas fueron me ignoran, hablan mal de mí y me ponen apodos, mientras que las femeninas eligieron me ponen apodos y hablan mal de mí.

Los agresores masculinos seleccionaron lo ignoro y les pongo apodos que ofenden, las agresoras eligieron lo ignoro.

\section{COMUNICACIÓN Y DEFENSA DE VÍCTIMAS}

La comunicación de los problemas fue mayormente con los amigos $74 \%$, y en menor grado con la familia $18 \%$, o guardaron los problemas para ellos $15 \%$.

Tabla 4. Percepción de manifestaciones de maltrato entre iguales ( $n=93)$.

\begin{tabular}{|c|c|c|c|c|c|c|}
\hline \multirow[t]{2}{*}{ Tipo de maltrato entre iguales } & \multicolumn{2}{|c|}{ Agresores } & \multicolumn{2}{|c|}{ Víctimas } & \multicolumn{2}{|c|}{ Testigos } \\
\hline & $\mathrm{n}^{\circ}$ & $\%$ & $\mathrm{n}^{\circ}$ & $\%$ & $\mathrm{n}^{\circ}$ & $\%$ \\
\hline Ignorar & 17 & 18.0 & 10 & 10,7 & 19 & 20,4 \\
\hline No dejar participar & 9 & 9,6 & 5 & 5,3 & 12 & 12,9 \\
\hline Insultar & 14 & 15,0 & 10 & 10,7 & 24 & 25,8 \\
\hline Poner apodos & 17 & 18,0 & 14 & 15,0 & 35 & 37,6 \\
\hline Hablar mal de él o ella & 19 & 20,4 & 17 & 18,0 & 35 & 37,6 \\
\hline Esconder cosas & 3 & 3,2 & 5 & 5,3 & 11 & 11,8 \\
\hline Romper cosas & 5 & 5,3 & 1 & 1,0 & 5 & 5,3 \\
\hline Robar cosas & 7 & 7,5 & 8 & 8,6 & 7 & 7,5 \\
\hline Pegar & 7 & 7,5 & 5 & 5,3 & 10 & 10,7 \\
\hline Amenazar para intimidar & 8 & 8,6 & 11 & 11,8 & 12 & 12,9 \\
\hline Acosar sexualmente & 4 & 4,3 & 2 & 2,1 & 6 & 6,4 \\
\hline Obligar a hacer cosas que no quiere & 4 & 4,3 & 3 & 3,2 & 6 & 6,4 \\
\hline Amenazar con arma & 1 & 0,1 & - & - & - & - \\
\hline
\end{tabular}


Tabla 5. Tipo de maltrato en función de actores y género de las víctimas $(n=93)$.

\begin{tabular}{|c|c|c|c|c|}
\hline \multirow[t]{2}{*}{ Víctimas } & \multicolumn{2}{|c|}{ Masculino (38) } & \multicolumn{2}{|c|}{ Femenino (37) } \\
\hline & $\mathrm{n}^{\circ}$ & $\%$ & $\mathrm{n}^{\circ}$ & $\%$ \\
\hline Me ignoran & 18 & 19,3 & 9 & 9,6 \\
\hline No me dejan participar & 7 & 7,5 & 8 & 8,6 \\
\hline Me insultan & 7 & 7,5 & 7 & 7,5 \\
\hline Me ponen apodos & 14 & 15,0 & 22 & 23,6 \\
\hline Hablan mal de mí & 14 & 15,0 & 16 & 17,2 \\
\hline Me obligan a hacer cosas que no quiero & 9 & 9,6 & 6 & 6,4 \\
\hline \multirow[t]{2}{*}{ Agresores } & \multicolumn{2}{|c|}{ Masculino (38) } & \multicolumn{2}{|c|}{ Femenino (37) } \\
\hline & $\mathrm{n}^{\circ}$ & $\%$ & $n^{\circ}$ & $\%$ \\
\hline Lo ignoro & 24 & 25,8 & 17 & 18,2 \\
\hline No lo dejo participar & 8 & 8,6 & 2 & 2,1 \\
\hline Lo insulto & 13 & 13,9 & 4 & 4,3 \\
\hline Le pongo apodos que ofenden & 15 & 16,1 & 4 & 4,3 \\
\hline Hablo mal de él o de ella & 10 & 10,7 & 8 & 8,6 \\
\hline
\end{tabular}

\section{PERCEPCIÓN DE CONDUCTA MALICIOSA O ACOSO SEXUAL}

\section{DISCUSIÓN}

La defensa de las víctimas expresadas como ayuda fue inferior al $50 \%$. La inacción y la indiferencia primaron alcanzando alrededor de 50\%.

El acoso sexual tuvo la misma frecuencia en la relación entre docentes y entre estudiantes, la conducta promiscua fue mayor entre los estudiantes, y fue similar la conducta maliciosa. El intercambio de recompensas por favores sexuales fue mayor entre profesores y estudiantes.

La Universidad, considerada como un centro de excelencia donde acuden los estudiantes en busca del conocimiento,

para debatir ideas y generar investigaciones que resuelvan los problemas de la sociedad, se ha visto afectada por la violencia y discriminación ${ }^{(9,19)}$.

La violencia es el uso de la fuerza para conseguir un fin, especialmente para dominar a alguien o imponer algo. La violencia institucional se da a todas las edades, desde la edad preescolar hasta en adultos universitarios ${ }^{(4-7,7,15)}$. Entre los estudiantes de medicina, se han descrito hechos de bullying entre pares y también de docentes hacia los estudiantes y viceversa $(8,10,12,13,16)$. La encuesta mide percepciones de los estudiantes y no hechos objetivables.

El abuso de autoridad se aprecia a veces en cada una de las acciones en los campos de ciencias básicas y de clínica, publicándose el maltrato verbal, físico, psicológico o sexual de docentes a estu-

Tabla 6. ¿Ha percibido algunas actividades institucionales relacionadas con acoso sexual? ( $n=93)$.

\begin{tabular}{lcccccc} 
Tipo de maltrato & \multicolumn{2}{c}{ Docentes } & & \multicolumn{2}{c}{ Estudiantes } \\
\cline { 2 - 3 } \cline { 5 - 6 } & $n^{\circ}$ & $\%$ & & $n^{\circ}$ & $\%$ \\
Acoso sexual & 10 & & 9,3 & & 11 & 10,7 \\
Intercambio de recompensas por favores sexuales & 4 & & 4,3 & & 2 & 2,15 \\
Conducta promiscua & 7 & & 7,5 & & 19 & 20,4 \\
Mostrar material sexual & 1 & & 1,0 & & 6 & 6,4 \\
Conducta maliciosa & 15 & & 16,1 & & 17 & 18,2 \\
\hline
\end{tabular}

diantes de medicina. Este abuso ha sido reportado como frecuente y perverso mayormente en los primeros años de estudio ${ }^{(10,11,20-24)}$. Las situaciones abusivas y hostiles minan el entusiasmo y desalientan al estudiante, son causantes de estrés y pueden contribuir a tornarlos apáticos, desmotivados y rudos. Los alumnos del último año relatan falta de respeto, de justicia y de responsabilidad de los educadores hacia los estudiantes, además de carencia de compromisos esenciales con el paciente ${ }^{(20)}$.

El bullying se manifiesta de diversas maneras, pero en el fondo siempre están el deseo de poder e implícitamente el hacer daño.

La agresión verbal es de uso frecuente alrededor del mundo entre los estudiantes como resultante del enojo motivado por los apodos, expresarse de mala manera, contestar de manera desafiante o realizar dibujos ofensivos. Esta manera de agresión verbal también se apreció en la conducta de docentes con estudiantes y Field lo señala como inadecuación e incompetencia del docente ${ }^{(25)}$. Similares resultados se han señalado en Colombia, Chile, Japón, EEUU, Finlandia, Australia ${ }^{(8,10,17,25)}$. El abuso verbal tiene que ser injurioso y repetido para recordar su relevancia ${ }^{(14)}$. En la Universidad de Oulu (Finlandia), se usan palabras injuriosas al dejar tareas de aprendizaje o investigación como castigo, por lo que se sugiere usar habilidades para promover ambientes seguros ${ }^{(5)}$.

La exclusión social es una condición con múltiples dimensiones, entre ellas la pobreza, etnia, raza, religión, segregación social, procedencia, tratamiento tradicional de 'cholo', hechos que los marginan y hacen vulnerables. Esta discriminación fue mayor entre estudiantes que en relación con docentes, evitando que participen en grupos e ignorando a los compañeros. En un estudio hecho en Panamá, las intimidaciones más comunes fueron la violencia oral y la discriminación ${ }^{(23)}$; similares resultados se han descrito en Colombia ${ }^{(8,26)}$. Este 
rechazo ha sido observado desde los primeros años de primaria ${ }^{(1,17,27,28)}$.

La agresión física entre estudiantes se caracteriza mayormente por empujones y golpes, esconder y apropiarse de algo; también se ha notado que los docentes no permiten el acceso a espacios y material de trabajo. En 1990, el pediatra Henry K. Silver fue el primero que documentó el maltrato al estudiante de Medicina indicando que el $46,4 \%$ de ellos había sido agredido en el pregrado alguna vez en la escuela de Medicina, y que cuando ellos fueron especialistas, esta cifra había ascendido a $80,6 \%{ }^{(29,30)}$.

El maltrato entre iguales (bullying) existe a nivel universitario, manifestándose a través de la agresión oral, física, psicológica y sexual. Los actores en este contexto son los agresores, las víctimas y los testigos. Los varones son más víctimas y agresores que las mujeres y estas le dan más importancia a ser testigos ${ }^{(8)}$.

Agresores: los estudiantes varones manifestaron que ignorar a los compañeros, poner apodos e insultarlos en términos que ofenden, así como hablar mal de él o ella era lo más frecuente. En las estudiantes, la conducta que predominó fue ignorar a los compañeros.

Víctimas: se quejaron de ser ignoradas y que se hablara mal de ellos o ellas. A su vez, las damas agregaron que les colocaban apodos con mayor frecuencia que a los varones. De manera similar a los estudios de Hoyos y Maida, las insultan, les esconden las cosas, les roban y les obligan a hacer cosas que no quieren ${ }^{(6,14)}$.

Testigos: no están implicados directamente en el bullying, pero solo brindaron ayuda un $42 \%$. Esto demuestra indiferencia y falta de solidaridad. En otras palabras: "Arréglatelas como puedas". Esta conducta se ha mencionado en educación primaria, secundaria y universitaria $^{(1-4,31)}$. En esta investigación, la calificación de las variables ha tenido mayores valores en los testigos que en agresores y víctimas.

Comunicación: los encuestados les cuentan sus problemas a los amigos, buscando apoyo en compañeros de su misma edad y grupo: tienen más confianza con ellos y en menor grado con sus familiares. Gómez hace referencia a este problema con la reflexión: "Hoy en día lamentablemente el padre de familia no participa activamente en el desarrollo y crecimiento de sus hijos" ${ }^{(31)}$. En general, la ayuda solidaria la recibieron en menos del $50 \%$ de las demandas ${ }^{(7)}$.

Conducta sexual: la sexualidad es parte importante de la personalidad y expresión de la conducta biopsicosocial que poseen todos los individuos a lo largo de su vida, e incluyen pensamientos, creencias, comportamientos, sentimientos, actitudes y valores que se manifiestan día a día en las relaciones entre compañeros en su entorno. La OMS nos recuerda que la salud sexual requiere un acercamiento respetuoso entre los individuos hacia la posibilidad de obtener placer y hacia las experiencias sexuales seguras, libres de coerción, discriminación y violencia, por lo que se debe considerar que las fuerzas sociales tienen un mayor impacto en el comportamiento sexual humano que los sistemas fisiológicos ${ }^{(32)}$.

La permisividad definida como "mayor liberalidad y aceptación en el juego sexual", más que en la penetración y el afecto, es parte de los procesos de cortejo y autonomía. Es una actitud flexible en presencia o ausencia de vinculación afectiva influida por principios morales, culturales, religiosos, usos o costumbres ${ }^{(33)}$

La conducta maliciosa y promiscua seguida del acoso entre estudiantes lideraron esta área. En la percepción de los alumnos, se encontró que en 9,3\% de los profesores primaron la conducta maliciosa y el acoso sexual. El intercambio de recompensas sexuales fue mayor entre docentes y alumnas que entre estudiantes ${ }^{(34)}$. Sin embargo, insistimos que nuestro estudio en esta área no es concluyente, por el empleo de una metodología subjetiva y de que debe ser reestudiada en el futuro. El acoso sexual también lo reportan otros estudios en nuestro país y el extranjero ${ }^{(9,10,14,29)}$.
En el último año de la carrera se evidencia un incremento notorio en la percepción de abuso sexual, lo que se podría explicar por el aumento del contacto individual docente-estudiante y las largas horas de trabajo propias del internado ${ }^{(13)}$. Además, se ha descrito que ese año es el de mayor riesgo con incremento de la ideación suicida, por lo que se sugiere, a nivel internacional, la implementación de programas de prevención (35).

Durante su formación universitaria, los estudiantes de Medicina están sometidos a diversos factores generadores de estrés que podrían llegar a ser adversos para su salud mental; muchos son inherentes a lo exigente y sacrificado de la misma formación, pero otros son potencialmente modificables por provenir de compañeros y sobre todo de profesores como parte de la interacción social ante figuras representativas de autoridad ${ }^{(6,19,24)}$. La importancia del estrés en la vida académica será motivo de una posterior publicación.

Existe abundante y creciente bibliografía en diversos países acerca de las nefastas consecuencias del maltrato hacia el estudiante de medicina ${ }^{(7-14)}$. Más que un asunto de evidencias, se trata de una cuestión de principios morales: uno de los pasos más importantes es reconocer su presencia, para luego hacer frente a las situaciones abusivas que son un problema; por tanto, es importante la Formación de Tutores con énfasis Académico y Humano ${ }^{(19)}$. Recordemos que los compromisos morales que sostienen los docentes con sus alumnos son medulares en la educación médica; ellos repercutirán en la relación que establezcan estos futuros profesionales con sus pacientes. Es urgente sensibilizar a nuestra comunidad sanfernandina y hacerla despertar de su letargo ante esta cruda realidad.

La Ética como disciplina filosófica que estudia el bien y el mal ha sido investigada de diversas maneras, pero el tema de bullying en la escuela de Medicina no ha sido o ha sido muy poco tratado en nuestro medio ${ }^{(9)}$. Esta in- 
vestigación ha demostrado su presencia con agresión física, psicológica, social y sexual al igual que en otros países, haciendo necesario cambiar los comportamientos de docentes y estudiantes, lo que se puede conseguir con el régimen tutorial señalado.

La investigación efectuada confirma los hallazgos encontrados en otros países sobre la existencia de violencia (bullying) en las escuelas de Medicina, que en ella participan docentes y alumnos, existiendo riesgo para la salud física y mental de los estudiantes, con obvia afectación del rendimiento académico. Se ha mencionado que la Formación de Tutores con énfasis Académico y Humano podría frenar o revertir la violencia en nuestra escuela de medicina.

Es conveniente señalar que una limitación del estudio es haberlo efectuado en base a percepciones, hecho subjetivo. Pero, nuestros hallazgos al encontrar indicadores de presencia de bullying en alumnos y docentes, exigen estudios más elaborados y con metodologías más precisas, ya que el problema de violencia institucional existe.

\section{REFERENCIAS BIBLIOGRÁFICAS}

1. Oliveros M, Figueroa L, Mayorga G, Cano B, Quispe $Y$, Barrientos A. Violencia escolar (bullying) en colegios estatales de primaria en el Perú. Rev peru pediatr. 2008;61(4):215-20

2. Oliveros M, Figueroa L, Mayorga G, Cano B, Quispe $Y$, Barrientos A. Intimidación en colegios estatales de secundaria del Perú. Rev peru pediatr. 2009;62(2):68-78.

3. Amemiya I, Oliveros M, Barrientos A. Condorimay $\mathrm{Y}$, Oliveros R, Ciberbullying en colegios privados y estatales de primaria en dos distritos de Lima Metropolitana. An Fac med. 2013;74(2):91-6.

4. Rautio A, Sunnari V, Nuutinen M, Laitala M. Mistreatment of university students most common during medical studies. BMC Medical Education. 2005;5(36):1-12.

5. Richman JA, Flaherty JA, Rospenda KM, Christensen ML. Mental health consequences and correlates of reported medical student abuse. JAMA. 1992;267:692-4
6. Hoyos de los Río O, Romero Santiago LM, Valega Mackenzie S, Molinares Brito C. El maltrato entre iguales por abuso de poder y exclusión social en estudiantes de una universidad privada de la ciudad de Barranquilla. Pensamiento Psicológico. 2009;6(13):109-26.

7. Paredes O, Sanabria-Ferrand PA, GonzálezQuevedo LA, Moreno Rehalpe SP, "Bullying" en las Facultades de Medicina Colombianas, mito o realidad. Rev Fac Med. 2010;18(2):161-72.

8. Moreno-Cubillas C, Sepúlveda E, Restrepo L. Discriminación y violencia de género Universidad de Caldas. Hacia la Promoción de la Salud. Ener-. junio 2012;17(1):59-76.

9. Munayco-Guillén F, Cámara-Reyes A, Muñoz-Tafur $L J$, Arroyo-Hernández $H$, Mejia CR, Lem-Arce $F$, Miranda Soberón UE. Características del maltrato hacia estudiantes de medicina de una universidad pública del Perú. Rev Peru Med Exp Salud Publica. 2016;33(1):58-66.

10. White GE. Sexual harassment during medical training: the perceptions of medical students at a university medical school in Australia. Med Educ. 2000;34:980-6.

11. Wood DF. Bullying and harassment in medical schools. Still rife and must be tackled. BMJ. 2006 Sept 30;333(7570):664-5. doi: 10.1136/ bmj.38954.568148.BE.

12. Quine L. Workplace bullying in junior doctors: questionnaire survey. BMJ. 2002;324(7342):8-9.

13. Daugherty SR, Baldwin DC Jr, Rowley BD. Learning, satisfaction and mistreatment during medical internship: A national survey of working conditions. JAMA. 1998;279:1194-9.

14. Maida AM, Vasquez A, Herskovic V, Calderon JL, Jacard M, Pereira A, Salinas LA. Percepción de conductas abusivas en estudiantes de medicina. Rev Méd Chile. 2006;134:1516-23.

15. Larsson C, Hensing G, Allbeck P. Sexual and gender-related harassment in medical education and research training: results from a Swedish survey. Med Educ. 2003;37:39-50.

16. Lebenthal A, Kaiserman I, Lernau O. Student abuse in medical school: a comparison of students' and faculty's perceptions. Isr J Med Sci. 1996;32:229-38.

17. Uhari M, Kokkonen J, Nuutinen M, Vainionpää L, Rantala H, Lautala P, Väyrynen M: Medical student abuse: An international phenomenon. JAMA. 1994;271:1049-51.

18. Cabezas C. Violencia escolar: El acoso del profesor hacia el alumno, 2008. Madrid. ISBN: 978-84-92539-19-2.

19. Perales A. Formación y capacitación de tutores en la Facultad de Medicina de la Universidad Nacional Mayor de San Marcos (2014-2015)

20. Rancich A, Donato M, Gelpi R. Incidentes moralmente incorrectos en la relación docente alumno en educación médica. Rev Argentina Cardiol. 2011;79(5):423-8.

21. Universidad Autónoma de Madrid, para el Informe Nacional del Defensor del Pueblo sobre Violencia Escolar (2000).
22. Castillo-Hernández M. Manifestaciones de la conducta de agresión en el contexto universitario. Escenarios. Enero-Junio de 2013;11(1):139-48.

23. Silva-Villarreal $S$, Castillo S, Vidal P, Mitre J, Quintero J. Prevalencia de violencia (bullying) en estudiantes de los ciclos básicos y preclínicos de la carrera de medicina de la Universidad de Panamá. iMed Pub Journals. 2013;9:4.1.

24. Del Toro AY, Gorguet M, Pérez Y, Ramos D. Estrés académico en estudiantes de medicina de primer año con bajo rendimiento escolar. MEDISAN. 2011;15(1):17.

25. Field T. Bullying in medicine. BMJ 2002;324(7340):786.

26. Moreno-Cubillas C, Sepúlveda-Gallego L. Discriminación y violencia con los estudiantes de medicina en la Universidad de Caldas. Inv Ed Med. 2013;2(1):37-41.

27. Ortega R, Monks C. Agresividad injustificada entre preescolares. Psicothema. 2005;17(3):453-8.

28. Garcia F, Sureta I, Monjas M. El rechazo entre iguales en la educación primaria. Anales de Psicología. Enero 2010;26(1):123-36.

29. Virtual Editor. To bully and be bullied: harassment and mistreatment in medical education. AMA J Ethics. 2014;16(3):155-60.

30. Silver HK. Medical student abuse: Incidence, severity, and significance. JAMA. 1990 Jan 28;263(4):527-32

31. Gómez Talavera LE. Nivel de agresión entre pares de estudiantes de una Universidad de Lima Metropolitana. Horiz Med. 2013;13(4):32-7.

32. Organización Mundial de la Salud (2002). Sexual and Reproductive Health. Disponible en linea: http://www.who.int/reproductive-health/gender/sexual_health.html\#2 (Recuperado Diciembre 2015.).

33. Cañizo E, Salinas F. Conductas sexuales alternas y permisividad en jóvenes universitarios. Enseñanza e investigación en Psicología. 2010;15(2):285-309.

34. Gutiérrez Morales I. Cyberbullying y sexing: percepción y propuestas de estudiantes universitarios. Multidisciplina. 2014;17:93-119.

35. Guille C, Zhao Z, Krystal J, Nichols B, Brady K, Sen S. Web-based cognitive behavioral therapy intervention for the prevention of suicidal ideation in medical interns a randomized clinical trial. JAMA Psychiatry. 2015 Dec;72(12):1192-8. doi: 10.1001/ jamapsychiatry.2015.1880.

Artículo recibido el 20 de mayo de 2016 y aceptado para publicación el 31 de junio de 2016.

Conflicto de interés: No existe en la presente investigación.

\section{Correspondencia:}

Dr. Miguel Oliveros Donohue

Correo electrónico: drmigueloliveros@hotmail.com 